\title{
Classification and Quantification of Occlusion Using Hidden Markov Model
}

\author{
C.R. Sahoo ${ }^{1}$, Shamik Sural ${ }^{1}$, Gerhard Rigoll ${ }^{2}$, and A. Sanchez ${ }^{3}$ \\ 1 School of Information Technology, Indian Institute of Technology, Kharagpur, India \\ rawchitta@gmail.com, shamik.sural@gmail.com \\ 2 Technical University of Munich, Munich, Germany \\ rigoll@tum.de \\ 3 Universidad Rey Juan Carlos, Madrid, Spain \\ angel.sanchez@urjc.es
}

\begin{abstract}
Over the last few years, gait recognition has become an active area of research. However, one of the shortcomings is lack of a method for quantifying occlusion in scenes used for capturing gait of individuals. Occlusion can occur primarily because of two reasons. Firstly, movement of certain body parts of a human being occludes some other body parts of the same human, which is called self occlusion and secondly, occlusion of the body parts caused by some other human being. The objective of this paper is to quantify occlusion of different parts of the human body using Hidden Markov Model (HMM) and classify the scene of occlusion as one of the three cases of occlusion, namely, self occlusion (single individual moving), occlusion in a crowd moving in same direction and occlusion due to movement of human beings approaching from opposite direction. We train one HMM for each body part relevant for gait recognition. An HMM is a statistical representation of probability distribution of a large number of possible sequences and in the current context these are the sequences of frames extracted at regular interval from a given video. The steps involved in achieving the objective are feature extraction, HMM training and finally the classification or hidden state generation.
\end{abstract}

Keywords: Occlusion, Gait Recognition, Body Part, HMM.

\section{Introduction}

One of the most active research areas in computer vision, human gait recognition broadly deals with detecting, tracking and identifying people from a sequence of images. Gait recognition has attracted great interest due to its promising applications in visual surveillance, with the added advantage of not requiring any active participation of the subject whose gait is under consideration. Even low end cameras with very low resolution provide adequate accuracy in recognition. However, occlusion handling still remains an area of concern [8].

Other than human gait recognition, a number of algorithms have been proposed for tracking and motion detection [9, 4. But none of them quantify occlusion. In this paper, we propose a method for quantifying occlusion by using 
HMM. HMM has been used successfully for speech recognition [5, 6] and handwritten word recognition [3]. The availability of sequential information in frames extracted from a video makes HMM an obvious choice for classifying the video into any desired class. Here we use HMM for classification of a given video into three classes based on the degree of occlusion. These classes are (i) self occlusion, i.e., a single human walking, (ii) occlusion in a crowd moving in same direction and (iii) occlusion due to movement of human beings approaching from opposite direction.

The primary aim of most of the researches on occlusion has been to identify occlusion while dealing with detection and tracking algorithms [4, 10, 7, 1]. Tracking methods can track individuals in image sequences, but real-world scenarios of crowded street scenes still pose a serious challenge for these methods because of occlusion during tracking. However, the focus on quantifying occlusion or to use occlusion as a tool has been negligible. We propose a method for quantifying occlusion and to use it for classification of the scene of occlusion. Until recently, the approximate reconstruction of scene of occlusion has been based on the knowledge of articulation. It is felt that knowledge of articulation along with the quantity of occlusion and scene of occlusion can result in a better approximation.

\section{Elements of the Proposed HMM}

States - A discrete HMM is characterized by a set of $N$ hidden states, a set of $M$ distinct observation symbols per state, state transition probability matrix $A=\left\{a_{i j}\right\}$, observation symbol generation probability matrix $B=\left\{b_{j}(\mathrm{k})\right\}$ and initial probability distibution matrix $\pi=\left\{\pi_{i}\right\}$ [5] .

Although states are hidden in an HMM, there is some physical significance attached to them. Moreover, the number of states also affects the cost of computation of the solutions to the problems addressed by HMM. The number of states and the number of HMMs required to achieve the overall objective are closely linked. Therefore, after careful consideration, we propose six HMMs, one for each body part, namely, two hands, two legs, one torso and one head.

For states of each HMM, we divide each body part into four segments, i.e., a band of $25 \%$ coverage vertically and consider occlusion of combinations of each

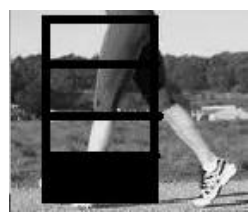

(a) 1 band: state $1-4$

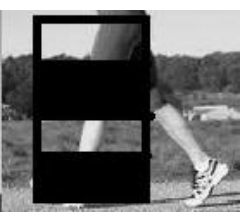

(b) 2 bands: state 5-10

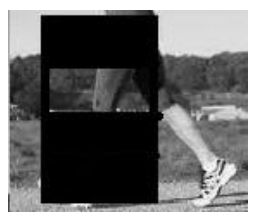

(c) 3 bands: state 11-14

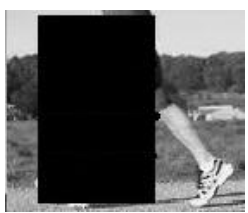

(d) 4 bands: state 15

Fig. 1. Different HMM states corresponding to Left Leg of a human being 
of these four bands as a state. Thus, at any given instant of time, the state can be occlusion of one of the four bands, or any combination of two bands out of four, or any combination of three bands out of four, or all the four bands or none of the four bands. Hence, the number of states $(N)$ is 16 . These states corresponding to occlusion of the left leg of a walking human being are explained with the help of images shown in Fig. 1. Similar state definitions are used for the other body parts.

For example let's consider the occlusion of left leg of a human being. We divide the leg into 4 segments and denote the occlusion of the lowest segment which includes foot, ankle and part of shinbone as state-1 and as we move up the leg, occlusion of the segments are numbered sequentially as state 2,3 and 4 . This is explained with the help of Fig. 1a where the segments are clearly marked, and state-1 is marked precisely. As explained above, Fig. 1a represents occlusion of one of the segments out of the four. We denote occlusion of combination of any 2 segments out of the 4 as states 5 to 10 , i.e, occlusion of segment- $1+$ segment- $2=$ state- 5 , segment- 1 + segment- $3=$ state- 6 and so on. This is explained with the help of Fig. 1b where state- 6 is marked precisely. Similarly, we denote occlusion of combination of any 3 segments as states 11 to 14 which is explained with the help of Fig. 1c where state-12 is marked precisely. We denote occlusion of combination of all the 4 segments as state- 15 which is explained with the help of Fig. 1d] and occlusion of none of the segments, i.e, when the entire leg is clearly visible as state- 16 .

Observation Symbol - For choosing observation symbol, color and shape information as proposed in 2 could be used. However, use of these observation symbols requires comparison with the original image, which may lead to false positives if the occluding object is of the same color or shape. We propose visibility of three reference points of different body parts as one observation vector for the HMM of that particular body part. These reference points are tabulated as follows -

\begin{tabular}{|l|l|l|l|}
\hline Body Part & Reference Point & Body Part & Reference Point \\
\hline \multirow{3}{*}{ Hand } & Shoulder Joint & \multirow{2}{*}{ Torso } & Neck \\
& Elbow & & Belly \\
& Finger Tip & & Lower Back \\
\hline \multirow{2}{*}{ Leg } & Hip Joint & \multirow{2}{*}{ Head } & Top of Head \\
& Knee & & Nose \\
& Tip of Toe & & Neck Joint \\
\hline
\end{tabular}

Corresponding to each reference point in each part, an observation vector is prepared depending on the visibility of the reference point. A '1' corresponds to visibility of that part and a '0' corresponds to lack of visibility of that part. For example, if the visibilities for Shoulder Joint, Elbow and Finger Tip corresponding to a hand at any time $t$ are respectively visible, not visible and visible, then the observation vector for that hand will be '101'. Different observation symbol vectors are explained with the help of images shown in Fig. 2 . 


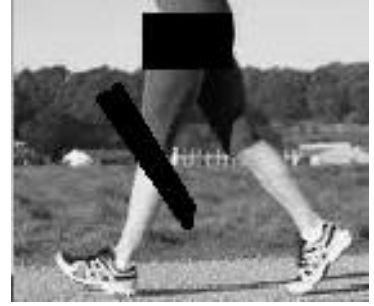

(a) Observation Symbol Vector '001'

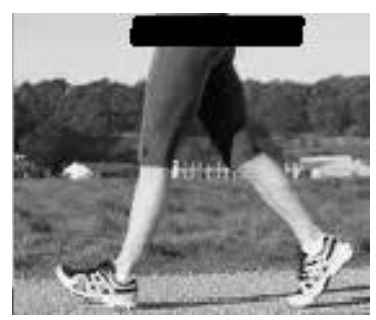

(b) Observation Symbol Vector '011'

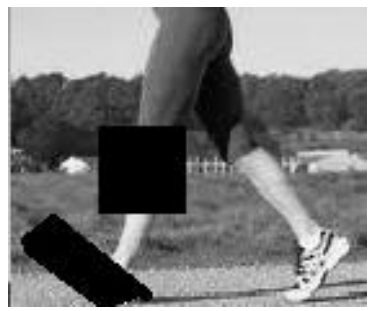

(c) Observation Symbol Vector '100'

Fig. 2. Different Observation Symbol Vectors for the Left Leg

Parameters - For determining initial parameters of an HMM, the following quantities are computed:

$$
a_{i j}=\frac{\text { No. of concurrent occurrences of state } S_{i} \text { at time t and state } S_{j} \text { at time t+1 }}{\text { No. of occurrences of state } S_{i} \text { for all time T }}
$$

$$
b_{j}(k)=\frac{\text { No. of concurrent occurrences of state } S_{j} \text { and observation symbol } v_{k}}{\text { No. of occurrences of state } S_{j}}
$$

Initially, we take all the states to be equiprobable and hence $\pi$ is a vector having $1 / 16$ as all its elements. For reestimating these parameters we follow the EM algorithm explained in [5].

\section{Experimental Setup}

In order to evaluate the feasibility of the proposed method we use Matlab for extracting frames from a video at regular intervals of time, extract the feature vectors, and use Kevin Murphy's HMM tool box for training HMMs for different parts of the body under different scenes of occlusion.

Training - For training the scene of crowd, a group of two, three, four and five persons were made to walk in a group and the feature vectors for the body parts of the second and the third person away from the camera were taken for training. The feature vectors for the body parts of the first person of some of the sequences were used to train the HMM for a single person walking. This was done because there is no occlusion between the camera and the first person and, hence, he can be treated as one possibly with only self occlusion. As the first step, every fifth frame from the video was extracted for HMM training.

For building the observation vector, the input was taken sequentially for the reference points of every frame where a left click was symbolized as ' 1 ' and a right 
click was symbolized as '0'. If a reference point was not visible, an approximate point was clicked where the reference point was expected to be present. This was done to input the coordinates of the particular body part which influences the extent of body part occluded, and determine the corresponding hidden state for training the HMM. The coordinates were taken by clicking at the extremities of occlusion of the body parts and the states were determined by comparing the extent of occlusion from the coordinates. In certain frames, some portions of the body parts were out of the frame, and were assumed to be occluded. Input for them could not be provided automatically since they were out of frame and hence the states for these frames were input manually.

Re-estimation of the parameters was done using five iterations of EM algorithm. Log likelihood of the model using the parameters after the fifth iteration was calculated and the parameters along with the log likelihood were saved.

Classification - Observation vector for the given video was built and log likelihood (LL) was calculated for all the three classes using the corresponding learnt parameters. The video was identified as the class with the closest LL.

Sequence Generation - For sequence generation, parameters of the identified class are taken and Veterbi algorithm as explained in [5] is used to trace back the hidden states.

\section{Experimental Results}

We performed experiments on video sequences recorded by our group. The classification results of eighteen sequences comprising of six sequences for each of the three classes are represented in the following confusion matrix.

\begin{tabular}{|c|c|c|c|}
\hline & Crowd & Opposite Direction & Single Individual \\
\hline Crowd & 6 & - & - \\
\hline Opposite Direction & 2 & 3 & 1 \\
\hline Single Individual & 1 & 2 & 3 \\
\hline
\end{tabular}

The rows of the above matrix represent the actual sequences which were used as input and the columns represent the output of the trained HMM. From the above matrix it can be seen that the classification for all the six crowd sequences given as input were classified correctly while out of the six sequences of people crossing from opposite direction three were correctly classified and of the six sequences of single individual again three were correctly classified.

For sequence generation, we take 20 frames from each of the 18 sequences. Out of the 18 sequences, 12 sequences had an accuracy of $75 \%$ or more, i.e., these sequences had at least 15 frames depicting correct state of occlusion.

\section{Conclusion}

Tracking of people has been the focus of most researchers working with gait as a biometric tool and approximate reconstruction of scene of occlusion with prior 
knowledge of articulation. But knowledge of articulation along with the quantity of occlusion and scene of occlusion can help in better approximation.

In this paper we have presented a scheme for classification of the type of occlusion and quantifying occlusion using hidden Markov model. It takes into account the visible parts of the human body and the states based on occlusion of a band of certain percentage of the body part. The accuracy of the model can be increased by refining the states to smaller bands, i.e., $5 \%$ or $10 \%$ of the body part. But in that case, the number of states and hence computing cost will increase. The observation symbol can also be further refined to be six reference points instead of three per body part. However, the number of training samples required for that will also go up.

\section{Acknowledgement}

This work is partially supported by Alexander von Humboldt Fellowship for Experienced Researchers.

\section{References}

1. Apostoloff, N., Fitzgibbon, A.: Learning spatiotemporal t-junctions for occlusion detection. In: IEEE Computer Society Conference on Computer Vision and Pattern Recognition, June 2005, vol. 2, pp. 553-559 (2005)

2. Marchesotti, L., Piva, S., Regazzoni, C.S.: A dynamic model integrating colour and shape information for objects tracking in conditions of occlusion. In: IEEE International Conference on Multimedia and Expo, June 2004, vol. 3, pp. 15471550 (2004)

3. Mohamed, M.A., Gader, P.: Generalized hidden markov models. ii. application to handwritten word recognition. IEEE Transactions on Fuzzy Systems 8(1), 82-94 (2000)

4. Pan, J., Hu, B.: Robust occlusion handling in object tracking. In: IEEE Conference on Computer Vision and Pattern Recognition, June 2007, pp. 1-8 (2007)

5. Rabiner, L.R.: A tutorial on hidden markov models and selected applications in speech recognition. Proceedings of the IEEE 77(2), 257-286 (1989)

6. Veeravalli, A.G., Pan, W.D., Adhami, R., Cox, P.G.: A tutorial on using hidden markov models for phoneme recognition. In: Proceedings of the Thirty-Seventh Southeastern Symposium on System Theory, March 2005, pp. 154-157 (2005)

7. Velipasalar, S., Wolf, W.: Multiple object tracking and occlusion handling by information exchange between uncalibrated cameras. In: IEEE International Conference on Image Processing, September 2005, vol. 2, pp. II-418-II-421 (2005)

8. Wang, L., Hu, W., Tan, T.: Recent developments in human motion analysis. Pattern Recognition 36(3), 585-601 (2003)

9. Wang, Y., Liu, Z., Zhou, L.: Learning hierarchical non-parametric hidden markov model of human motion. In: Proceedings of International Conference on Machine Learning and Cybernetics, August 2005, vol. 6, pp. 3315-3320 (2005)

10. Yilmaz, A., Li, X., Shah, M.: Contour-based object tracking with occlusion handling in video acquired using mobile cameras. IEEE Transactions on Pattern Analysis and Machine Intelligence 26(11), 1531-1536 (2004) 